\title{
Aprendizaje colaborativo, mediado por internet, en procesos de educación superior
}

\author{
Collaborative Learning in Higher Education Processes Mediated by Internet
}

\section{Processos de aprendizagem colaborativa no Ensino Superior mediados pela Internet}

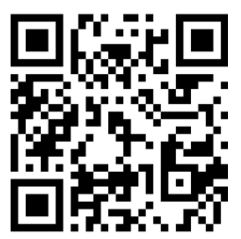

\section{Resumen:}

Introducción. El estudio del aprendizaje colaborativo mediado por internet se popularizó a partir de los años 90, cuando el uso de las mediaciones tecnológicas soportadas por internet evidenció que se podría aprender colaborando con la mediación del computador. Los desarrollos posteriores revisaron cómo las tecnologías favorecían los procesos de aprendizaje y de qué manera la colaboración podía ser incorporada en la educación en la modalidad virtual. Objetivo. Establecer líneas de estudio no exploradas que pueden contribuir al desarrollo de posteriores trabajos sobre el aprendizaje colaborativo en procesos de educación superior mediado por internet. Análisis. Se analizaron 225 documentos publicados en revistas indexadas en SCOPUS entre 1995 y 2018. Con la ayuda del paquete bibliometrix en el entorno R se estimó el comportamiento de las publicaciones en la franja de tiempo señalada, las áreas del conocimiento en las que más se publica sobre el tema, los documentos más relevantes por su número de citaciones, así como los énfasis temáticos asumidos en los estudios analizados, mediante redes de coocurrencia de términos clave definidos por las autorías. Resultados. Los resultados reflejan dispersión en la investigación sobre el tema, presumiblemente por la tendencia a desarrollar estudios aislados por disciplinas, orientados en su mayoría al análisis de la efectividad de herramientas tecnológicas y de entornos para favorecer el aprendizaje, sobre los resultados individuales, más no los grupales. Conclusiones. El aprendizaje colaborativo mediado por internet requiere ser estudiado en estudios interdisciplinares, además necesita ser estructurado, diseñado y enseñado en los programas de educación superior, ya que las herramientas tecnológicas son apoyos que median en el proceso de aprendizaje colaborativo, no son el fin del proceso educativo.

Palabras claves: Aprendizaje colaborativo; educación superior; educación universitaria; educación virtual; estrategias educativas. 
http://doi.org/10.15359/ree.25-2.23

http://www.una.ac.cr/educare

educare@una.ac.cr

\begin{abstract}
Introduction. The study of collaborative learning mediated by the Internet became popular in the 1990s when the use of technological mediations, supported by the Internet, showed that learning could be possible by collaborating with the computer's mediation. Later developments reviewed how technologies favored learning processes and how collaboration could be incorporated into virtual mode education. Objective. To establish unexplored study lines that can contribute to further works on collaborative learning in higher education processes mediated through the Internet. Analysis. It was conducted on 225 documents published in journals indexed in SCOPUS between 1995 and 2018. The use of the bibliometrix package in the R environment help to analyze the behavior of the publications in the indicated time frame, the areas of knowledge in which more is published about the topic, the most relevant documents due to their number of citations, and the thematic emphasis assumed in the studies analyzed; the analysis was carried out through networks of co-occurrence of key terms defined by the authors. Results. The results reflect dispersion in the research on the subject, presumably due to the tendency to develop isolated studies by disciplines, mostly oriented to analyzing the effectiveness of technological tools and environments to favor learning, on individual results but not group ones. Conclusions. Collaborative learning mediated through the Internet requires to be studied in interdisciplinary studies. It also needs to be structured, designed, and taught in higher education programs since technological tools are the support of the collaborative learning process, not the end of the educational process.
\end{abstract}

Keywords: Collaborative learning; higher education; university education; virtual education; educational strategies

Introdução. $O$ estudo da aprendizagem colaborativa mediada pela internet popularizou-se a partir dos anos 90, quando o uso de mediações tecnológicas, apoiadas pela internet, mostraram que ela poderia ser aprendida colaborando com a mediação do computador. Desenvolvimentos posteriores revisaram como as tecnologias favoreciam os processos de aprendizagem e como a colaboração poderia ser incorporada à educação no modo virtual. Objetivo. Estabelecer linhas de estudo inexploradas que possam contribuir para o desenvolvimento de trabalhos subsequentes sobre aprendizagem colaborativa em processos de ensino superior mediados pela Internet. Análise. Foram analisados 225 documentos publicados em periódicos indexados no SCOPUS entre 1995 e 2018. Com o auxílio do pacote bibliometrix no ambiente R, estimou-se o comportamento das publicações no intervalo de tempo indicado, áreas do conhecimento em que mais existem publicação sobre o tema, os documentos mais relevantes pelo número de citações, bem como pela ênfase temática assumida nos estudos analisados, por meio de redes de coincidências de termos-chave definidos pelos autores. Resultados. Os resultados refletem dispersão nas pesquisas sobre o tema, provavelmente devido à tendência de desenvolver estudos isolados por disciplinas, em sua maioria voltados para a análise da eficácia de ferramentas e ambientes tecnológicos para favorecer a aprendizagem, em resultados individuais, mas não em grupos. Conclusões. A aprendizagem colaborativa mediada pela Internet requer um estudo interdisciplinar, mas também precisa ser estruturada, desenhada e ministrada em programas de Ensino Superior, uma vez que as ferramentas tecnológicas são suportes que medeiam o processo de aprendizagem colaborativa, e não o fim do processo educacional.

Palavras-chave: Aprendizagem colaborativa, ensino superior, educação universitária, educação virtual, estratégias educacionais 


\section{Introducción}

Uno de los principales retos de la educación superior tiene que ver con la formación de profesionales que posean habilidades correspondientes a las necesidades de la sociedad actual. De acuerdo con Voogt y Roblin (2012), las habilidades que demanda el siglo XXI poseen dos características: transversalidad y multidimensionalidad. La transversalidad indica que dichas competencias no están directamente vinculadas a un campo específico, pero tienen incidencia en varios campos e interactúan simultáneamente con otras. La multidimensionalidad se relaciona con conocimientos, habilidades y actitudes que le permiten al individuo resolver problemas complejos en situaciones impredecibles. Aprender a colaborar es una de esas habilidades transversales, exigidas por la dinámica de la época contemporánea (Ahmadi y Besançon, 2017). Colaborar implica el trabajo que dos o más personas llevan a cabo juntas, en busca de un mismo fin, con criterios flexibles y de respeto (linuma et al., 2016).

La enseñanza y el aprendizaje de la colaboración es un reto para la enseñanza en todos los niveles, principalmente en la educación superior, si se tiene en cuenta que es en estos contextos en donde se prepara para el mundo laboral a profesionales de las diferentes áreas. Por esta razón resulta fundamental comprender de qué se trata aprender colaborativamente, cuáles son sus principales fundamentos, de qué manera se ha estudiado este constructo en la educación superior mediada por internet y cuáles son los caminos inexplorados que requieren ser abordados para contribuir al avance de este campo de estudio.

Con respecto al origen, puede decirse que el estudio sobre el aprendizaje colaborativo se remonta a los años 1970 y 1980. En principio, las investigaciones analizaron exclusivamente la dinámica de pequeños grupos y la interacción estudiantil cara a cara en grupos de educación primaria. Posteriormente, fueron desarrolladas en grupos de estudiantes de educación secundaria y universitaria, gracias a la evidencia de investigación en educación disponible sobre buenas prácticas en los procesos de enseñanza (Bradford y Peck, 1997) y la efectividad para la instrucción de grupos pequeños (Johnson y Johnson, 2009). De hecho, de acuerdo con Chickering y Gamson (1987), ese fue el punto de partida que dio lugar a la generación de directrices para el diseño y desarrollo de los procesos de enseñanza en la educación superior, dentro de los cuales se planteó la interacción entre estudiantes en términos cooperativos.

El planteamiento teórico que soporta la perspectiva del aprendizaje cooperativo es el denominado histórico cultural desarrollado por Vygotsky. Desde este enfoque teórico, la interacción de los factores interpersonales (sociales), los histórico culturales, así como los individuales en el desarrollo humano son fundamentales para el aprendizaje (Schunk, 2012). Dada esta característica, se dice que en la colaboración se presenta una interdependencia 
http://doi.org/10.15359/ree.25-2.23

http://www.una.ac.cr/educare

educare@una.ac.cr

entre miembros de un grupo que aprende conjuntamente. Dillenbourg (1999) le denomina interdependencia social. La interdependencia social se manifiesta cuando las personas integrantes de los equipos de trabajo perciben que el logro de sus metas puede darse si, y solo si, sus compañeros también logran las suyas (Johnson y Johnson, 2004). El desarrollo de la interdependencia social durante el aprendizaje colaborativo se ha estudiado principalmente con grupos de estudiantes de la modalidad presencial (Vega Vaca et al., 2013), ya que la interacción cara a cara posibilita que dicha interdependencia se desarrolle.

De otra parte, Strijbos y Fischer (2007) refieren que el aprendizaje colaborativo es un tema de estudio abordado por disciplinas como la psicología, las ciencias de la computación, las ciencias de negocios, la ingeniería y, por supuesto, la educación; se ha analizado desde diferentes vertientes, por ejemplo: adquisición de conocimiento (cuando el estudiantado aprende con otro), tipos y niveles de participación de los involucrados en el aprendizaje (definición de roles y características de desempeño en ellos y herramientas de mediación) y generación de conocimiento (efectos en el logro). En este sentido, el estudio del aprendizaje colaborativo no es exclusivo de una única disciplina, ni de una única modalidad educativa.

Por esto, a partir de la vertiginosa incursión de las tecnologías de la información y la comunicación (TIC) a partir de los años 90, la investigación en el campo asumió una identidad propia (al emerger de la simbiosis de TIC y aprendizaje colaborativo) conocida como Computer Supported Collaborative Learning ( $\mathrm{CSCL}$, por sus siglas en inglés) (García Chitiva y Suárez Guerrero, 2019).

A la luz de este constructo, los estudios de sobre el aprendizaje con otros individuos ha tenido diferentes énfasis. Por ejemplo, se han examinado las interacciones asincrónicas y sincrónicas entre estudiantes que aprenden colaborativamente en la plataforma Blackboard (Suárez Guerrero, 2010), en modalidades mixtas o B-learning (Han y Ellis, 2019), en modalidades de aprendizaje en cursos masivos y abiertos (MOOCS, por sus siglas en inglés) (Weinhardt y Sitzmann, 2019). Algunos de esos trabajos han analizado los procesos de construcción y negociación compartida de conocimiento, mediante las interacciones escritas en foros (Castellanos Ramírez y Niño (2018). También, se han analizado las interacciones mediante el trabajo colaborativo mediado por Wikis (Aydın y Yildiz ,2014) y otros estudios han abarcado los tipos de metodologías propicias para facilitar el aprendizaje (la metodología cooperativa), así como el empleo de herramientas pertinentes para favorecer el proceso de enseñanza aprendizaje (Flores et al., 2011). Es decir, el aprendizaje colaborativo es un tema central en la investigación relacionada con la educación virtual, principalmente en educación superior (Mercado Borja et al., 2019). 
Teniendo en cuenta la relevancia del aprendizaje colaborativo mediado por las tecnologías soportadas por internet, es preciso platearse la pregunta: ¿Qué se sabe sobre los estudios recientes acerca aprendizaje colaborativo en educación superior mediado por internet?

De acuerdo con Chen et al. (2018), la investigación sobre aprendizaje colaborativo ha involucrado tanto uso de tecnologías, como el de estrategias de soporte para el proceso de aprendizaje. Mediante un meta-análisis, este autor examinó 425 estudios a partir de los cuales comparó el tamaño del efecto del uso de tecnologías o de estrategias de soporte para el proceso de aprendizaje, en relación con tres aspectos: 1) El efecto positivo de la colaboración sobre en el conocimiento obtenido, las habilidades obtenidas y en la percepción estudiantil sobre el trabajo colaborativo mediado por computador e internet, 2) El efecto positivo del uso del computador y el internet en la adquisición de conocimientos y 3) el efecto del uso de entornos o herramientas de aprendizaje adicionales sobre la adquisición de conocimientos de sus participantes. En todos los casos, Chen et al. (2018) identificó que fueron comparados grupos expuestos a los tres elementos o condiciones mencionadas, con otros grupos de iguales características (tamaño de muestra, niveles de formación, mismo contenido de aprendizaje) que no fueron expuestos a dichas condiciones. Como producto de este meta-análisis, este autor estableció que los estudios revisados analizaron el aprendizaje colaborativo mediado por computadore internet con aspectos de la dimensión social (interacciones con los compañeros y compañeras, trabajo en equipo), con objetivos cognitivos (logro de aprendizaje en un contenido, desarrollo de habilidades en resolución de problemas), aspectos interpersonales (percepción sobre la motivación y gusto por el trabajo en colaboración) y, concluyó que, en general, los resultados estuvieron más enfocados a los procesos de aprendizaje que al proceso de colaboración en sí mismo.

Dado lo expuesto anteriormente, es claro que el aporte de los estudios sobre el tema se ha centrado en los procesos de aprendizaje y el efecto del uso de herramientas tecnológicas y metodologías, más no en cómo potenciar, por ejemplo, el proceso de aprender a colaborar y fortalecer así las habilidades colaborativas del estudiantado en los contextos de educación superior mediados por internet. Por lo tanto, el objetivo de este trabajo es establecer líneas de estudio no exploradas que pueden contribuir al desarrollo de posteriores trabajos en este campo de estudio. Para ello, se realizó un análisis bibliométrico de 225 documentos que examinaron el aprendizaje colaborativo en procesos de educación superior mediado por internet. En lo que sigue, se presentan el método y los materiales en donde se explican los detalles de la recuperación, procesamiento y análisis bibliométrico de los documentos analizados. Posteriormente se presentan los resultados, la discusión y las conclusiones. 
http://doi.org/10.15359/ree.25-2.23

http://www.una.ac.cr/educare

educare@una.ac.cr

\section{Método}

\section{Tipo de estudio}

Este trabajo sigue las especificaciones de un estudio bibliométrico (Salas et al., 2018). Los estudios bibliométricos, según Aria y Cuccurullo (2017), son particularmente útiles para complementar la revisión de estudios de un tema, al estructurar y cuantificar la información bibliográfica con el propósito de identificar los países, autores y autoras, instituciones más influyentes en la comunidad científica, los tópicos investigados hasta el momento, los cambios en los límites temáticos de las disciplinas y encontrar tendencias temporales de investigación, entre otros aspectos.

\section{Procedimiento de búsqueda de documentos y de análisis de datos}

Se utilizó la base de datos SCOPUS como fuente de información primaria para la consulta de los registros bibliográficos que incluyeran las palabras clave Collaborative learning, internet y university en el título, el abstract o las palabras clave de cada trabajo publicado por fuentes indexadas a SCOPUS, con el siguiente query string TITLE-ABS-KEY ("Collaborative learning" AND "internet" AND "university"). Se obtuvo un total de 225 trabajos publicados entre 1995 y 2018 por parte de 170 fuentes que incluían artículos publicados en revistas, artículos en prensa, libros, capítulos de libro, artículos de conferencias, notas, editoriales y revisiones. Para realizar el análisis de los datos se emplearon técnicas de estadística descriptiva y visualización de datos en R (Wickham y Grolemund, 2017). La tasa de crecimiento promedio anual de publicaciones se calculó con el algoritmo annualgrowthrate implementado en el paquete bibliometrix de Aria y Cuccurullo (2017). Además, se analizó la productividad de los autores y autoras, los niveles de colaboración y los temas más dominantes, a través de técnicas de visualización en redes (Cherven, 2015) generadas en bibliometrix. Igualmente se usaron los mapas de estructuras conceptuales yel algoritmo KeywordGrowth, implementados y desarrollados a través del paquete bibliometrix, para establecer cuáles han sido los temas más dominantes en las investigaciones a lo largo de la última década. El algoritmo KeywordGrowth genera el ranking de palabras clave más frecuentemente mencionadas por los documentos analizados para establecer el estado de la investigación, en general, sobre el tema. 
http://doi.org/10.15359/ree.25-2.23

\section{Resultados}

La Figura 1 muestra que el crecimiento promedio interanual de la publicación sobre aprendizaje colaborativo en entornos virtuales de aprendizaje (EVA) en educación superior fue de 13,43\%. Entre 1995 y 2007, la comunidad científica en el área produjo el 31\% de las publicaciones, mientras que 69\% restante entre 2008 y 2018, lo cual muestra que la mayor cantidad de contribuciones ocurrieron en la última década. La Figura 2 ilustra que, en las áreas de ciencias sociales, ciencias de la computación e ingeniería se concentra la mayor cantidad de producción científica sobre aprendizaje colaborativo en EVA en educación superior. Curiosamente, disciplinas más afines al tema, tales como psicología y educación, muestran menores volúmenes de publicación.

Figura 1: Registro histórico de publicaciones sobre Aprendizaje Colaborativo en EVA en Educación superior

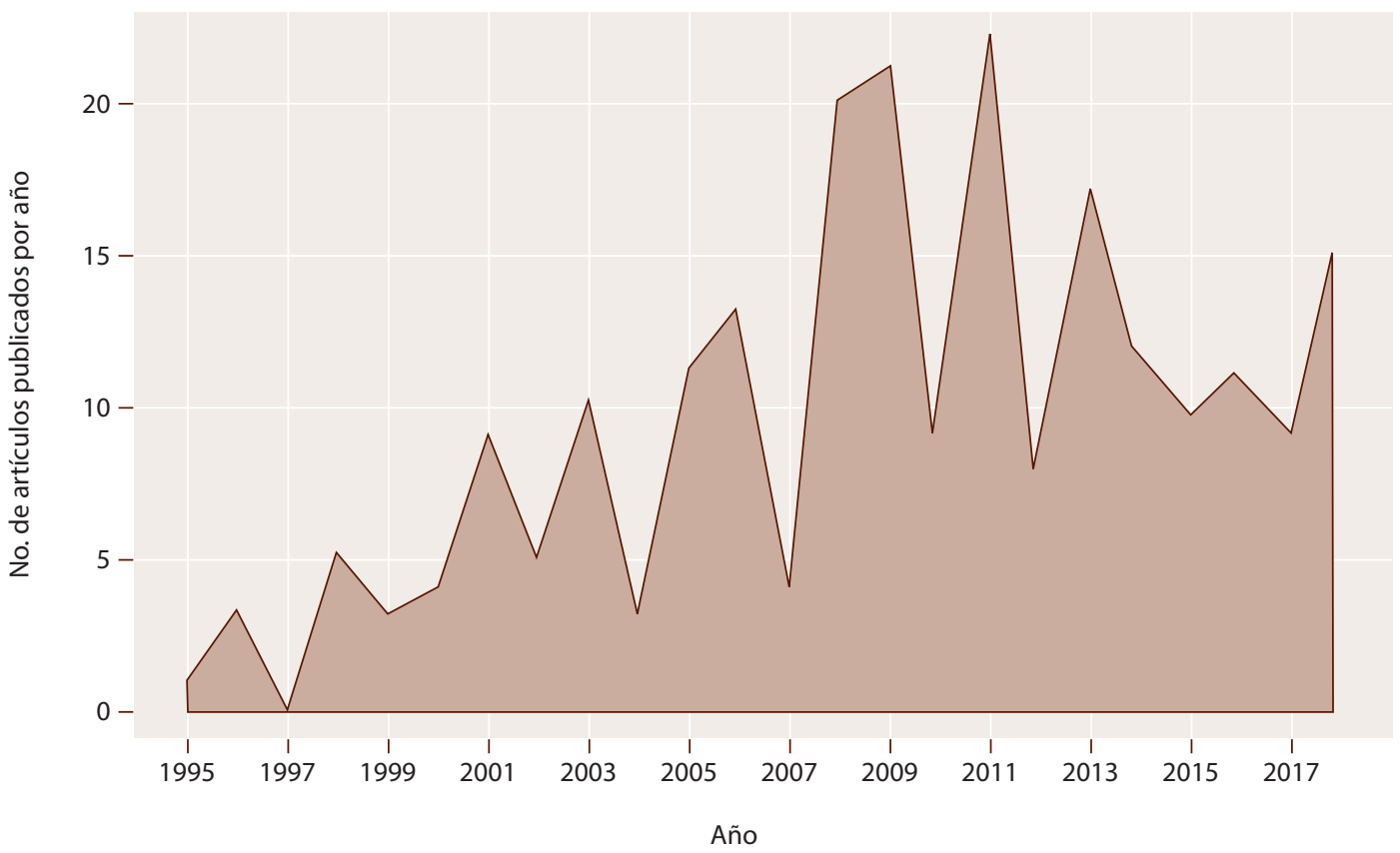

Nota: Elaboración propia. 
http://doi.org/10.15359/ree.25-2.23

http://www.una.ac.cr/educare

educare@una.ac.cr

Figura 2: Distribución del volumen de publicaciones por disciplina científica

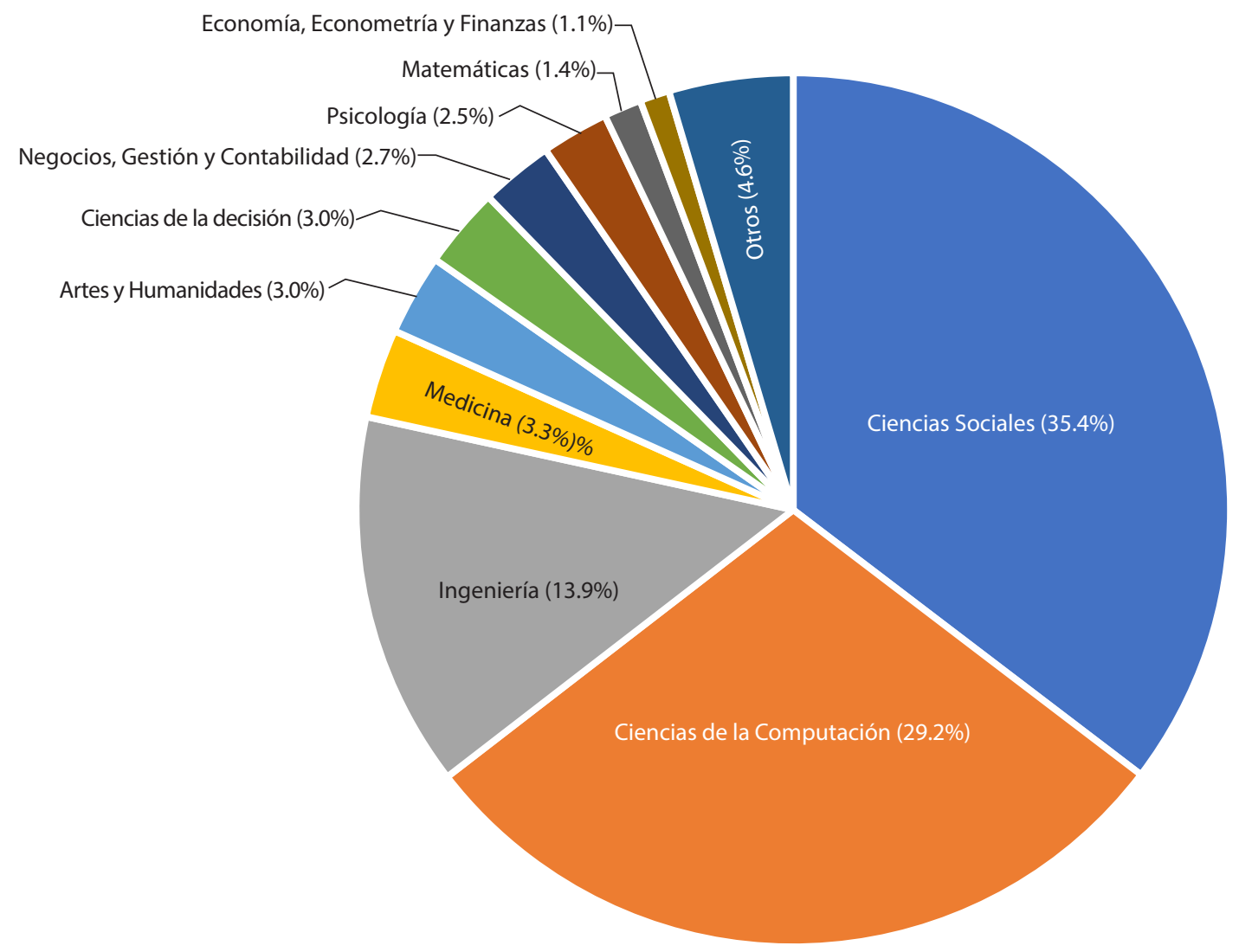

Nota: Elaboración propia.

La Figura 3 ilustra la presencia de seis grupos de tópicos identificados a través de una red de coocurrencia de palabras clave. La conexión entre algunas de estas palabras con una línea recta representa la coocurrencia sistemática de estos términos en las publicaciones. Es claro que, en general, los temas lucen poco conectados entre sí, lo cual muestra un panorama poco cohesionado entre sus temáticas. 
http://doi.org/10.15359/ree.25-2.23

http://www.una.ac.cr/educare educare@una.ac.cr

Figura 3: Red de coocurrencia de palabras clave de los trabajos publicados sobre Aprendizaje colaborativo en

EVA en Educación superior

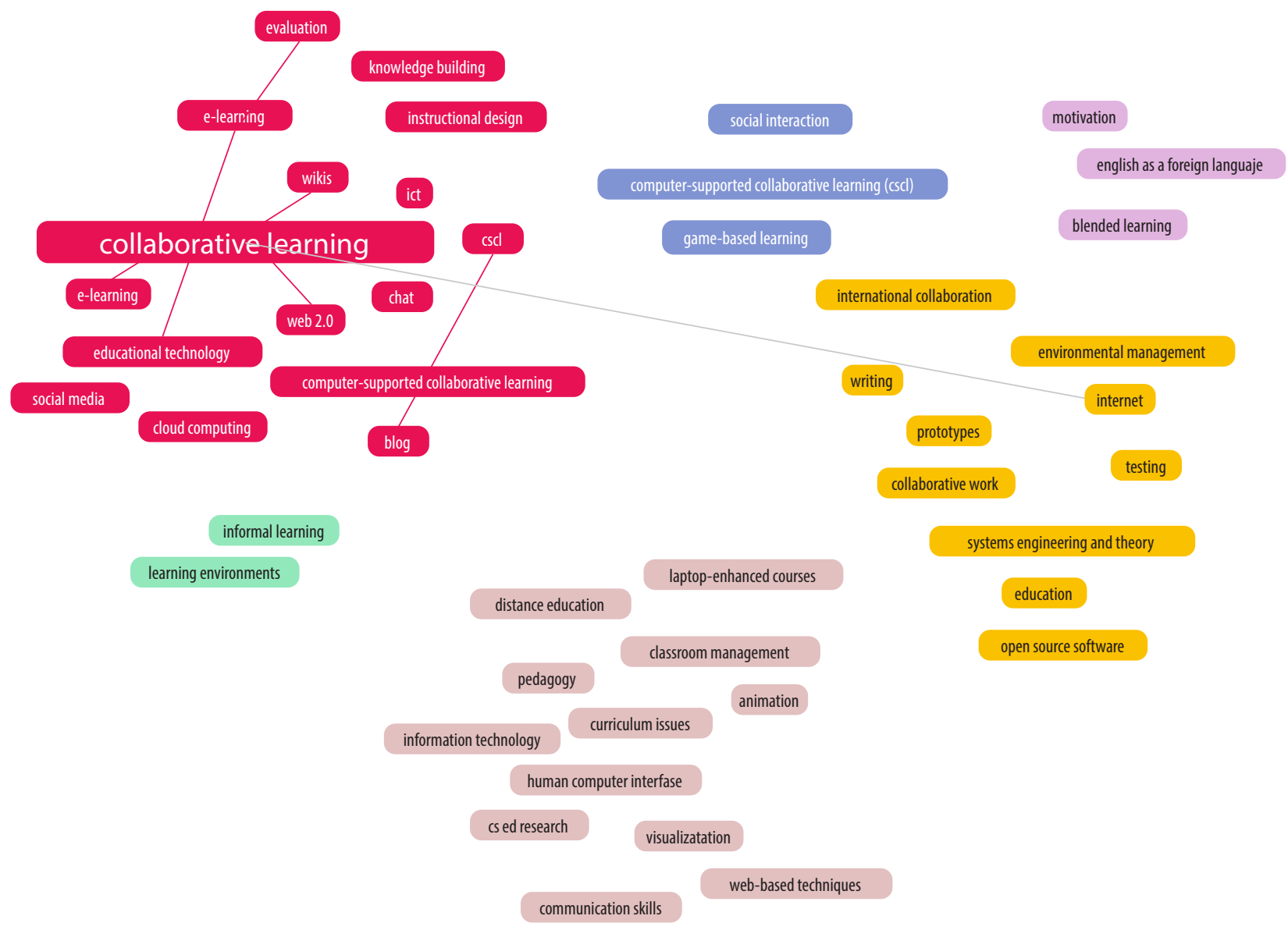

Nota: Elaboración propia.

El grupo dominante en esta red (en cuadros rojos) está representado por los trabajos que destacan como palabras clave el aprendizaje colaborativo, el e-learning, la evaluación, la tecnología educativa, el web 2.0, las wikis, el diseño instruccional, la construcción de conocimiento, las redes sociales, blog, chat, cloud computing y aprendizaje colaborativo apoyado por computadoras o cscl (por sus siglas en inglés).

El segundo grupo (en cuadros amarillos) aglutina los trabajos con la palabra clave internet que se muestra asociada de forma visible con aprendizaje colaborativo, es decir, hay presencia de lo que, en la teoría de grafos se conoce como centralidad intermedia. De acuerdo con Cherven (2015), la centralidad intermedia explica el puente o conexión que se establece entre dos nodos 
http://doi.org/10.15359/ree.25-2.23

http://www.una.ac.cr/educare

educare@una.ac.cr

que pertenecen a dos grupos diferentes (aprendizaje colaborativo e internet) de dos regiones de la red que, de otra manera no se hubiesen conectado. Esta conexión refleja el uso frecuente, en conjunto, que se ha dado a estos dos términos en los estudios analizados. Igualmente, se aprecia que en este segundo grupo destacan términos no directamente vinculados con los EVA en la educación superior, sino con otras disciplinas como la gerencia (e.g., trabajo colaborativo, gerencia ambiental, colaboración internacional) o la ingeniería (e.g., teoría e ingeniería de sistemas, prototipo, software de fuente abierta, escritura de códigos).

El tercer grupo en la red (en cuadros marrones) agrupa términos que sí están mucho más vinculados al quehacer cotidiano de la educación superior, ya que las palabras clave más empleadas son educación a distancia, pedagogía, manejo del aula, cursos basados en portátiles, habilidades de comunicación, tecnologías de información, etc.

El cuarto grupo (en recuadros azules) integra los trabajos orientados a la interacción social y el aprendizaje colaborativo apoyado en computador y basado en juegos.

El quinto grupo (en recuadros morados) reúne estudios orientados a la motivación, al inglés como segunda lengua y al aprendizaje mixto. El último grupo (en recuadros verdes) integra trabajos sobre entornos de aprendizaje y aprendizaje informal.

Las agrupaciones presentes en estos cluster de palabras evidencian un marcado enfoque hacia el uso de tecnologías soportadas por internet (plataformas, redes sociales), que contribuyen en los procesos de aprendizaje colaborativo en entornos virtuales y permiten comprender cómo interactúa el estudiantado cuando aprende en trabajo conjunto. El resto de las agrupaciones, de manera general, refleja trabajo aislado por áreas como la ingeniería o el inglés $y$, el uso de herramientas tecnologías y estrategias que favorezcan el aprendizaje, en entornos formales e informales.

De otra parte, con respecto a las fuentes, las temáticas anteriores han sido publicadas en diferentes fuentes disciplinares, algunas de las cuales con alto impacto científico, según su $\mathrm{H}$-index. Así pues, se observaron publicaciones en revistas como Computers and Education (H-index = 134), Computers in Human Behavior (H-index =123), International Review of Research in Open and Distance Learning $(\mathrm{H}$-index $=50)$, o actas de conferencias tales como Conference Proceedings of the American Society of Engineering Education ( $\mathrm{H}$-Index = 27), Conference Proceedings of the Association for Computing Machinery $(\mathrm{H}-$ Index $=83)$, Advances in Information and Communication Technology de la International Federation for Information Processing $(\mathrm{H}$-Index $=40)$. A pesar del impacto científico de las fuentes en donde se han publicado trabajos de investigación relacionados con el aprendizaje colaborativo en EVA en educación superior, cabe resaltar que el número de publicaciones no excede los cuatro artículos por fuente, lo cual revela que no existe un área que se perfile como la preponderante sobre el tema. 
http://doi.org/10.15359/ree.25-2.23

Los 20 artículos con mayor número de citas aparecen en la Figura 4. Estos estudios abordan siete temáticas específicas: 1) Diseño de ambientes virtuales de aprendizaje (de Lucia et al., 2009). 2) Diseño de metodologías y diseño de herramientas para favorecer el aprendizaje y la colaboración (Sancho-Thomas et al., 2009). 3) Análisis y evaluación del efecto de metodología de aprendizaje colaborativo (Brahimi y Sarirete, 2015). 4) Análisis y evaluación de entornos y ambientes virtuales de aprendizaje propicios para el aprendizaje Brett y Nagra, 2005; Edirisingha et al., 2009). 5) Análisis del efecto del uso de herramientas web sobre el aprendizaje, la colaboración (de aprendizaje entre estudiantes y de colaboración científica) y la comunicación (Campbell y Pargas, 2003; Cho y Lee, 2008; Padilla-Meléndez et al. 2008; Regueras et al., 2009; Romano et al., 2011; Sendall et al., 2008; Wilkerson et al., 2005). 6) Implicaciones para el aprendizaje de otras metodologías (competitiva) en contraste con la colaborativa, en ambientes b-learning (HoicBozic et al., 2009). 7) Evaluación del uso y el efecto positivo para el aprendizaje y la colaboración de herramientas móviles y redes sociales (Chang et al., 2009; Na et al., 2008).

Figura 4: Lista de artículos con mayor número de citas recibidas según las métricas de SCOPUS

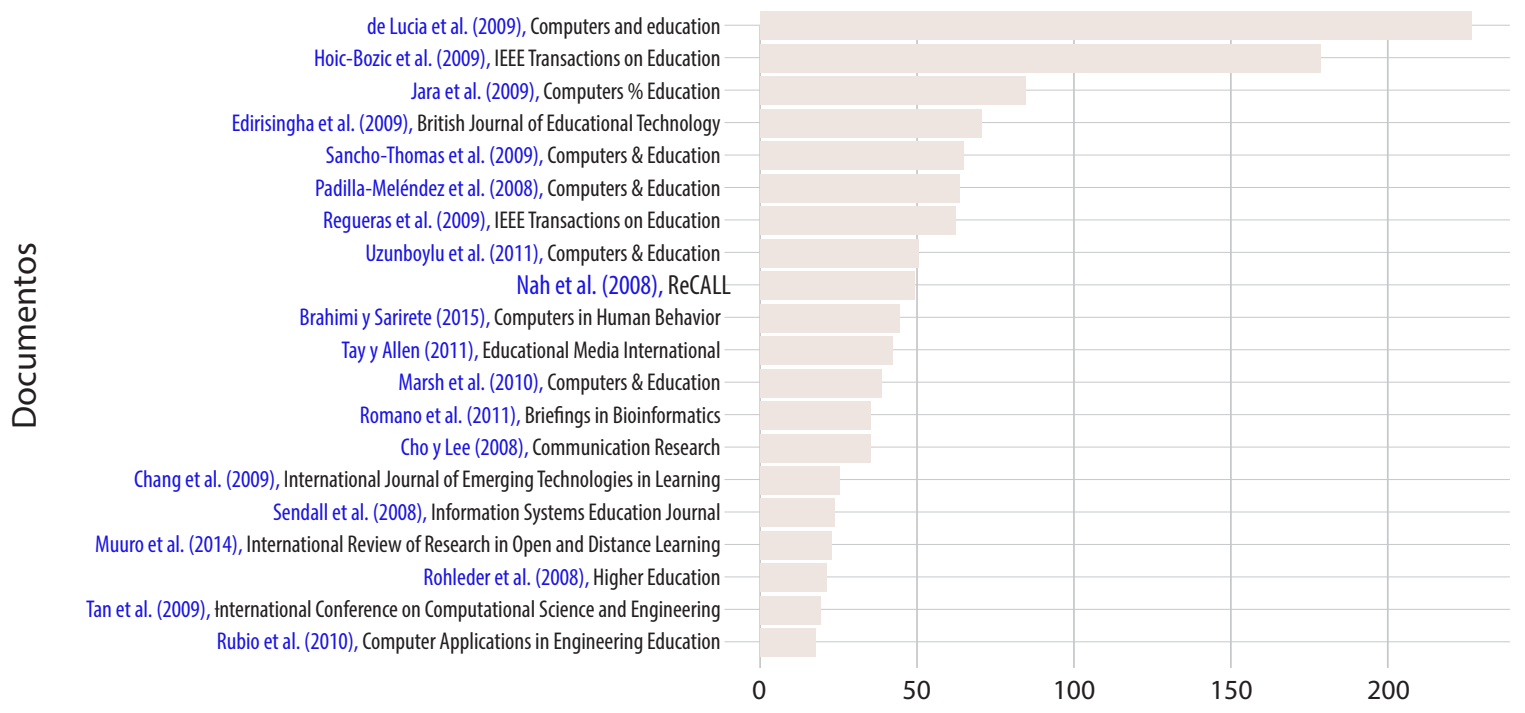

Número total de citas

Nota: Elaboración propia.

\section{Discusión}

Establecer líneas de estudio no exploradas que pueden contribuir al desarrollo de posteriores trabajos en este campo de estudio fue el objetivo del presente trabajo. La incursión del internet en la educación superior permitió transformar paradigmas frente a las posibilidades 
http://doi.org/10.15359/ree.25-2.23

http://www.una.ac.cr/educare

educare@una.ac.cr

de aprender y enseñar. A medida que las tecnologías de la información continúan avanzando y se generan dispositivos inteligentes más sofisticados, el aprendizaje mediado por tecnologías basada en internet adquiere mayor relevancia como modalidad de aprendizaje (Wang, 2019) e igualmente el aprendizaje colaborativo como una metodología efectiva para favorecerlo.

Los resultados indicaron que la investigación sobre el tema está dispersa en diferentes áreas (e.g., ciencias de la computación, ciencias sociales, medicina, artes y humanidades, etc). Se advierte que dicha dispersión refleja la tendencia por desarrollar investigación aplicada para establecer qué condiciones metodológicas, de entorno de aprendizaje y cuáles herramientas tecnológicas (incorporadas en los EVA, redes sociales, aplicaciones web y de celulares) favorecen el aprendizaje, la comunicación y la colaboración entre estudiantado universitario. Esto explica la desconexión entre los tópicos de los trabajos analizados reflejada en el análisis de redes de palabras clave. En contraste, en el grupo de documentos analizados no existen estudios destacados que conceptualicen ni analicen el proceso estructural, en sí, de la colaboración en el contexto de aprendizaje mediado por el internet.

Si bien existen trabajos previos que consideran y proponen comprensiones estructurales del aprendizaje colaborativo, tal como los de Dillenbourg (1999) (dos o más personas, aprenden juntos comunes) y Kreijns et al. (2002) (coopresencia, conciencia, comunicación, colaboración y coordinación), el énfasis de los estudios analizados refleja que se sigue dando prioridad a elementos individuales de los grupos aprendices y a aspectos externos del aprendizaje grupal. De esta manera, resulta notorio que se pierde de vista que el proceso natural de aprendizaje en colaboración implica, además de la mediación tecnológica, procesos cognitivos, de interacción, de negociación de significados y de construcción grupal. Esta circunstancia se refleja en la red de términos clave. Este aspecto coincide con lo señalado por Kreijns et al. (2003), quien afirma que la enseñanza-aprendizaje de la colaboración requiere de estructuras definidas en las que se diseñe, intencionalmente, la instrucción de cómo deben desarrollarse las interacciones durante la colaboración, pues de otra manera no sucederán por la simple designación de los grupos de trabajo.

De otra parte, con respecto a los resultados de las fuentes más relevantes, se evidencia que existe una preocupación importante por analizar y estudiar los procesos de aprendizaje colaborativo mediado por internet en la educación superior. No obstante, se encuentra que sigue siendo un trabajo aislado, por disciplinas, lo que explica por qué 225 artículos se encuentran publicados en 170 fuentes de información diferentes. Esta evidencia presente en la bibliografía que aborda el tema sugiere que aún el estudio de la colaboración en procesos de aprendizaje mediados por internet se encuentra en un proceso exploratorio, particularmente centrado en analizar el efecto de las herramientas tecnológicas sobre variables asociadas al desempeño y la relación de los procesos de aprendizaje colaborativos con habilidades personales e intrapersonales. En este caso, lo esencial no sería contar con una única fuente de publicación disciplinar, pero sí, con trabajos que revelen colaboraciones interdisciplinares 
que permitan analizar los componentes estructurales de la colaboración en relación con otras variables y que permitan comprender de qué se trata el proceso de colaboración y cómo puede ser orientado y desarrollado para desplegar y formar actitudes y habilidades de colaboración entre estudiantes de educación superior.

Finalmente, aunque los estudios analizan la colaboración en grupos de estudiantes, estos generalmente examinan los resultados desde los desempeños y percepciones individuales y no grupales. Vale la pena mencionar, aquí, que, si bien el estudio de la colaboración no ha analizado la articulación con otros constructos que investigan el trabajo colaborativo desde denominaciones diferentes, por ejemplo, el sistema de memoria transactiva (TMS) desarrollado por Wegner (1987) y abordado por Hollingshead (2001), Bodemer y Dehler (2011) y Jackson y Moreland (2009), desde este constructo de TMS sí se han analizado los procesos de colaboración, desde una vertiente cognitiva (García-Chitiva, 2020). En dicho enfoque, se analiza el funcionamiento de aprendizaje y trabajo grupal-colaborativo, en tres dimensiones de análisis: experticia, credibilidad y coordinación (Lewis, 2003, 2004). La articulación de estudios que examinen, de manera conjunta, el aprendizaje colaborativo mediado por internet y el sistema de memoria transactiva pueden generar resultados promisorios que den más pistas para conocer el funcionamiento al interior de los grupos colaborativos y, en esa medida, puedan dar nuevas rutas de estudio en el campo.

\section{Conclusiones}

1. Aprender a colaborar es una de las habilidades necesarias para desempeñarse en las esferas sociales contemporáneas. Por ello, es preciso que las instituciones de educación superior, particularmente aquellas que ofertan programas mediados por internet o con la modalidad virtual, estructuren e incorporen, dentro de sus currículos, la formación en habilidades de colaboración. Debe superarse la comprensión desdibujada del aprendizaje y trabajo colaborativo como una simple reunión de integrantes de un equipo en el desarrollo de una tarea en común. El aprendizaje colaborativo pone en sintonía a miembros de equipos que comparten metas de aprendizaje y trabajo comunes, que negocian significados, construyen temas de memoria transactiva compartida que les permite desarrollar de manera democrática y especializada las asignaciones y tareas emprendidas.

2. Las herramientas tecnológicas soportadas por internet son apoyos que median en el proceso de aprendizaje colaborativo, no deben ser considerados el fin del proceso educativo, pues estas esencialmente son mediaciones que permiten aprender en equipo, cuando no comparten espacios presenciales en tiempo sincrónico. 
http://doi.org/10.15359/ree.25-2.23

http://www.una.ac.cr/educare

educare@una.ac.cr

3. El estudio del aprendizaje colaborativo mediado por internet requiere de trabajos interdisciplinares que visibilicen la articulación de constructos afines abordados y de amplio en otras disciplinas, ya que esto permite evitar la dispersión de los hallazgos y, en cambio, proveer aportes de mayor rigor, en este caso, al estudio del aprendizaje colaborativo mediado por internet o colaboración en entornos virtuales de aprendizaje.

4. Dado que la pandemia del COVID- 19 evidenció que una contingencia puede volcar los procesos educativos a metodologías exclusivamente virtuales, se hace necesario reflexionar y tomar acciones de transformación y mejora para que el aprendizaje mediado por las tecnologías soportadas por internet cuente con diseños y orientaciones pedagógicas estructuradas que preparen a profesionales en formación y a estudiantes en general, para saber cómo trabajar en equipos eficientes y efectivos.

\section{Recomendaciones}

Para futuros estudios que empleen la metodología de análisis bibliométrico, se sugiere que se exploren otras alternativas de análisis complementario, que usen, por ejemplo, matrices descriptivas de los documentos para contar con detalles más amplios, de los contextos de las tareas, muestras e instrumentos empleados.

También, resultaría oportuno poder analizar los estudios que reposan en las fuentes más citadas que en este estudio fueron reportadas como destacadas, para poder identificar también líneas de trabajo en relación con el aprendizaje colaborativo, que puedan resultar útiles para ser exploradas en entornos multidisciplinares.

\section{Declaración de procedencia}

Este artículo es parte del desarrollo conceptual de la tesis doctoral del autor en el Doctorado Interinstitucional en Educación de la Universidad Pedagógica Nacional, Bogotá, Colombia.

\section{Declaración de Material complementario}

Este artículo tiene disponible, como material complementario:

-La versión preprint del artículo en https://doi.org/10.5281/zenodo.4414899

\section{Referencias}

Ahmadi, N. y Besançn, M. (2017). Creativity as a stepping stone towards developing other competencies in classrooms. Education Research International, 1-9. https://doi. org/10.1155/2017/1357456 
http://doi.org/10.15359/ree.25-2.23

Aria, M. y Cuccurullo, C. (2017). Bibliometrix: An R-Tool for comprehensive science mapping analysis. Journal of Informetrics, 11(4), 959-975. https://doi.org/10.1016/j.joi.2017.08.007

Aydın, Z. y Yildiz, S. (2014). Using wikis to promote collaborative ELF writing. Language Learning \& Technology, 18(1), 160-180. https://www.lltjournal.org/item/2845

Bodemer, D. y Dehler, J. (2011). Group awareness in CSCL environments. Computers in Human Behavior, 27(3), 1043-1045. https://doi.org/10.1016/j.chb.2010.07.014

Bradford, B. M. y Peck, M. W., Jr. (1997). Achieving AECC outcomes through the seven principles for good practice in undergraduate education. Journal of Education for Business, 72(6), 364368. https://doi.org/10.1080/08832323.1997.10116872

Brahimi, T. y Sarirete, A. (2015). Learning outside the classroom through MOOCs. Computers in Human Behavior, 51, 604-609. https://doi.org/10.1016/j.chb.2015.03.013

Brett, P. y Nagra, J. (2005). An investigation into students' use of a computer-based social learning space: Lessons for facilitating collaborative approaches to learning. British Journal of Educational Technology, 36(2), 281-292. https://doi.org/10.1111/j.1467-8535.2005.00457.x

Campbell, A. B y Pargas, R. P. (2003). Laptops in the classroom. ACM SIGCSE Bulletin, 35(1), 98-102. https://doi.org/10.1145/792548.611942

Castellanos Ramírez, J. C. y Niño, S. A. (2018). Aprendizaje colaborativo y fases de construcción compartida del conocimiento en entornos tecnológicos de comunicación asincróna. Innovación Educativa, 18(76), 69-88. http://www.scielo.org.mx/scielo.php?pid=S166526732018000100069\&script $=$ sci arttext

Chang, V., Gütl, C., Kopeinik, S. y Williams, R. (2009). Evaluation of collaborative learning settings in 3D virtual worlds. International Journal of Emerging Technologies in Learning, 4, 6-17. https://doi.org/10.3991/ijet.v4s3.1112

Chen, J., Wang, M., Kirschner, P. A. y Tsai, C.-C. (2018). The role of collaboration, computer use, learning environments, and supporting strategies in CSCL: A meta-analysis. Review of Educational Research, 88(6), 799-843. https://doi.org/10.3102/0034654318791584

Cherven, K. (2015). Mastering gephi network visualization. Packt Publishing.

Chickering, A. W. y Gamson, Z. F. (1987). Seven principles for good practice in undergraduate education. AAHE Bulletin, 3-6. https://files.eric.ed.gov/fulltext/ED282491.pdf

Cho, H. y Lee, J.-S. (2008). Collaborative information seeking in intercultural computer-mediated communication groups:Testing the influence of social context using social networkanalysis. Communication Research, 35(4), 548-573. https://doi.org/10.1177/0093650208315982 
http://doi.org/10.15359/ree.25-2.23

http://www.una.ac.cr/educare

educare@una.ac.cr

De Lucia, A., Francese, R., Passero, I. y Tortora, G. (2009). Development and evaluation of a virtual campus on Second Life: The case of SecondDMI. Computers \& Education, 52(1), 220-233. https://doi.org/10.1016/j.compedu.2008.08.001

Dillenbourg, P. (1999). What do you mean by collaborative learning? En P. Dillenbourg (Ed.), Collaborative learning: Cognitive and computational approaches (pp. 1-19). Elsevier.

Edirisingha, P., Nie, M., Pluciennik, M. y Young, R. (2009). Socialisation for learning at a distance in a 3-D multi-user virtual environment. British Journal of Educational Technology, 40(3), 458479. https://doi.org/10.1111/j.1467-8535.2009.00962.x

Flores, O., Verdú, N., Giménez, P., Juárez, J., Mur, J. A. y Menduiña, C. (2011). Web 2.0 en la docencia universitaria: Aprendizaje colaborativo a través de la tecnología. Electronic Journal of Research in Educational Psychology, 9(24), 931-960. http://ojs.ual.es/ojs/index.php/EJREP/ article/view/1480

García-Chitiva, M. del P. (2020). Implications of Transactive Memory Systems (TMS) for higher education. Revista Espacios, 41(46), 338-351. https://www.revistaespacios.com/ a20v41n46/a20v41n46p28.pdf

García-Chitiva, M. del P. y Suárez-Guerrero, C. (2019). Estado de la investigación sobre la colaboración en entornos virtuales de aprendizaje. Píxel-BIT Revista de Medios y Educación, 56, 169-191. https://doi.org/10.12795/pixelbit.2019.i56.09

Han, F. y Ellis, R. A. (2019). Identifying consistent patterns of quality learning discussions in blended learning. The Internet and Higher Education, 40, 12-19. https://doi.org/10.1016/j. iheduc.2018.09.002

Hoic-Bozic, N., Mornar, V. y Boticki, I. (2009). A blended learning approach to course design and implementation. IEEE Transactions on Education, 52(1), 19-30. https://doi.org/10.1109/ $\underline{\text { TE.2007.914945 }}$

Hollingshead, A. B. (2001). Cognitive interdependence and convergent expectations in transactive memory. Journal of Personality and Social Psychology, 81(6), 1080-1089. https:// doi.org/10.1037/0022-3514.81.6.1080

linuma, M., Matsuhashi, T., Nakamura, T. y Chiyokura, H. (2016). Student awareness change in computer supported collaborative learning (CSCL) environment. International Journal of Information and Education Technology, 6(6), 448-452. https://doi.org/10.7763/IJIET.2016. $\underline{\mathrm{V} 6.730}$

Jackson, M., y Moreland, R. L. (2009). Transactive Memory in the Classroom. Small Group Research, 40(5), 508-534. https://doi.org/10.1177/1046496409340703 
Jara, C. A., Candelas, F. A., Torres, F., Dormido S., Esquembre, F. y Reinoso, O. (2009). Real-time collaboration of virtual laboratories through the internet. Computers \& Education, 52(1), 126-140. https://doi.org/10.1016/j.compedu.2008.07.007

Johnson, D. W. y Johnson, R. T. (2004). Cooperation and the use of technology. En D. H. Jonassen (Ed.), Handbook of research on educational communications and technology (2. ${ }^{\text {a }}$ ed., pp. 785-810). Asociación for Educational Communications and Technology.

Johnson, D. W. y Johnson, R. T. (2009). An educational psychology success story: Social interdependence theory and cooperative learning. Educational Researcher, 38(5), 365-379. https://doi.org/10.3102/0013189X09339057

Kreijns, K., Kirschner, P. A. y Jochems, W. (2002). The sociability of computer-supported collaborative learning environments. Educational Technology \& Society, 5(1), 8-22. https:// www.jstor.org/stable/jeductechsoci.5.1.8? seq=1\&cid=pdf-reference\#references tab contents

Kreijns, K., Kirschner, P. A. y Jochems, W. (2003). Identifying the pitfalls for social interaction in computer-supported collaborative learning environments: A review of the research. Computers in Human Behavior, 19(3), 335-353. https://doi.org/10.1016/S0747$\underline{5632(02) 00057-2}$

Lewis, K. (2003). Measuring transactive memory systems in the field: Scale development and validation. Journal of Applied Psychology, 88(4), 587-604. https://doi.org/10.1037/0021$\underline{9010.88 .4 .587}$

Lewis, K. (2004). Knowledge and performance in knowledge-worker teams: A longitudinal study of transactive memory systems. Management Science, 50(11), 1519-1533. https:// doi.org/10.1287/mnsc.1040.0257

Marsh B., Mitchell, N. y Adamczyk, P. (2010). Interactive video technology: Enhancing professional learning in initial teacher education. Computers \& Education, 54(3), 742-748. https://doi. org/10.1016/j.compedu.2009.09.011

Mercado Borja, W., Guarnieri, G. y Luján Rodríguez, G. (2019). Análisis y evaluación de procesos de interactividad en entornos virtuales de aprendizaje. Trilogía Ciencia Tecnología Sociedad, 11(20), 63-99. https://doi.org/10.22430/21457778.1213

Muuro M. E., Wagacha W. P., Oboko R. y Kihoro, J. (2014). Students' perceived challenges in an online collaborative learning environment: A case of higher learning institutions in Nairobi, Kenya. International Review of Research in Open and Distance Learning, 15(6), 132161. 10.19173/irrodl.v15i6.1768

Nah, K. C., White, P. y Sussex, R. (2008). The potential of using a mobile phone to access the internet for learning EFL listening skills within a korean context. ReCALL, 20(3), 331-347. https://doi.org/10.1017/S0958344008000633 
http://doi.org/10.15359/ree.25-2.23

http://www.una.ac.cr/educare

educare@una.ac.cr

Padilla-Meléndez, A., Garrido-Moreno, A. y del Aguila-Obra, A. R. (2008). Factors affecting e-collaboration technology use among management students. Computers \& Education, 51(2), 609-623. https://doi.org/10.1016/j.compedu.2007.06.013

Regueras, L. M., Verdú, E., Muñoz, M. F., Perez, M. A., de Castro, J. P. y Verdú, M. J. (2009). Effects of competitive e-learning tools on higher education students: A case study. IEEE Transactions on Education, 52(2), 279-285. https://doi.org/10.1109/TE.2008.928198

Rohleder P., Bozalek, V., Carolissen R., Leibowitz, B. y Swartz, L. (2008). Students' evaluations of the use of e-learning in a collaborative project between two south african universities. Higher Education, 56(1), 95-107. 10.1007/S10734-007-9091-3

Romano, P., Giugno, R. y Pulvirenti, A. (2011). Tools and collaborative environments for bioinformatics research. Briefings in Bioinformatics, 12(6), 549-561. https://doi.org/10.1093/ $\underline{\text { bib/bbr055 }}$

Rubio, R., Martín, S. y Morán, S. (2010). Collaborative web learning tools: Wikis and blogs. Computer Applications in Engineering Education, 18(3), 502-511. https://doi.org/10.1002/ cae.20218

Salas, G., Ravelo-Contreras, E. L., Mejía, S., Andrades, R., Acuña, E., Espinoza, F., Nuñez, M., Barboza-Palomino, M., Ventura-León, J., Caycho-Rodríguez, T.y Pérez-Acosta, A. (2018). Dos décadas de Acta Colombiana de Psicología: Un análisis bibliométrico. Acta Colombiana de Psicología, 21(2), 13-25. https://doi.org/10.14718/ACP.2018.21.2.2

Sancho-Thomas, P., Fuentes-Fernández, R. y Fernández-Manjón, B. (2009). Learning teamwork skills in university programming courses. Computers \& Education, 53(2), 517-531. https:// doi.org/10.1016/j.compedu.2009.03.010

Schunk, D. H. (2012). Teorías del aprendizaje. Una perspectiva educativa (6. ${ }^{a}$ ed.). Pearson Educación.

Sendall, P., Ceccucci, W. y Peslak, A. R. (2008). Web 2.0 Matters: An analysis of implementing Web 2.0 in the classroom. Information Systems Education Journal, 6(64), 1-17. http://isedj. org $/ 6 / 64$ /

Strijbos, J.-W. y Fischer, F. (2007). Methodological challenges for collaborative learning research. Learning and Instruction, 17(4), 389-393. https://doi.org/10.1016/j.learninstruc.2007.03.004

Suárez Guerrero, C. (2010). A prendizaje cooperativo e interacción asíncrona textual en contextos educativos virtuales. Pixel-Bit. Revista de Medios y Educación, 36, 53-67. http://hdl.handle. net/11441/22608

Sung, Y.-T., Yang, J.-M. y Lee, H.-Y. (2017). The effects of mobile-computer-supported collaborative learning: Meta-analysis and critical synthesis. Review of educational Research, 87(4), 768805. https://doi.org/10.3102/0034654317704307 
Tan, Q., Kinshuk, Kuo, Y.-H., Jeng, Y.-L., Wu, P.-H., Huang, Y.-M., Liu, T.-C. y Chang, M. (2009). Location-based adaptive mobile learning research framework and topics. International Conference on Computational Science and Engineering (pp. 140-147). 10.1109/CSE.2009.96

Tay, E. y Allen, M. (2011). Designing social media into university learning: Technology of collaboration or collaboration for technology? Educational Media International, 48(3), 151 163. https://doi.org/10.1080/09523987.2011.607319

Uzumboylu, H., Bicen, H. y Cavus, N. (2011). The efficient virtual learning environment: A case study of web 2.0 tools and windows live spaces. Computers \& Education, 56(3), 720-726. https://doi.org/10.1016/j.compedu.2010.10.014

Vega Vaca, M. L., Vidal Rodríguez, D. y García, M. del P. (2013). Avances acerca de los efectos del aprendizaje cooperativo sobre el logro académico y las habilidades sociales en relación con el estilo cognitivo. Revista Colombiana de Educación, 64, 155-174. https://doi.org/10.1 7227/01203916.64rce155.174

Voogt, J. y Roblin, N. P. (2012). A comparative analysis of international frameworks for $21^{\text {st }}$ century competences: Implications for national curriculum policies. Journal of Curriculum Studies, 44(3), 299-321. https://doi.org/10.1080/00220272.2012.668938

Wang, J. (2019). Application of blending learning based on network learning space in teaching design of digital art. International Journal of Emerging Technologies in Learning, 14(3), 177189. https://doi.org/10.3991/ijet.v14i03.10107

Wegner, D. M. (1987). Transactive memory: A contemporary analysis of the group mind. En B. Mullen y G. R. Goethals (Eds.), Theories of Group Behavior (pp. 185-208). Springer. https:// doi.org/10.1007/978-1-4612-4634-3 9

Wegner, D. M. (1995). A computer network model of human transactive memory. Journal of Chemical Information and Modeling, 13(3), 319-339. https://scholar.harvard.edu/dwegner/ publications/computer-network-model-human-transactive-memory

Weinhardt, J. M. y Sitzmann, T. (2019). Revolutionizing training and education? Three questions regarding massive open online courses (MOOCs). Human Resource Management Review, 29(2), 218-225. https://doi.org/10.1016/j.hrmr.2018.06.004

Wickham, H. y Grolemund, G. (2017). R for data science. O’Reilly. http://r4ds.had.co.nz/

Wilkerson, M., Griswold, W. G. y Simon, B. (2005). Ubiquitous presenter: Increasing student access and control in a digital lecturing environment. ACM SIGCSE Bulletin, 37(1), 116-120. https:// doi.org/10.1145/1047344.1047394 